\title{
Table of the First Moment of Ranked Extremes
}

\author{
Julius Lieblein ${ }^{\star}$ and Herbert E. Salzer ${ }^{\star \star}$
}

\begin{abstract}
Let a sample in $n$ independent random values from the extreme-value distribution, with c.d.f. $\Phi(y)=\exp \left(-e^{-y}\right)$, be arranged in decreasing order and denoted by $y_{1}, y_{2}$, $y_{m}, . \quad ., y_{n}$. The table gives the expected values for all these order statistics for sample size not exceeding 25. For the larger samples, up to $n=100$, the expected values are given only for the first 26 largest values.
\end{abstract}

\section{Explanation and Use of Table}

Table 1, prepared at the suggestion of Bradford F. Kimball, gives the expected values, $E\left(y_{m}\right)$, of order statistics from the type I distribution of "reduced" largest values, $\operatorname{Prob}\{Y \leq y\}=\Phi(y)=\exp \left(-e^{-y}\right)$. This distribution was discussed in considerable detail in a series of lectures by E. J. Gumbel [1].' ${ }^{1}$ The entries were computed from the formula given by B. F. Kimball [2],

$E\left(y_{m}\right)=C+\sum_{t=0}^{m-1}(-1)^{t}\left(\begin{array}{c}n \\ t\end{array}\right) \Delta^{t} \ln (n-t)$,

$$
m=1,2, \ldots ., n,
$$

where

$$
y_{1} \geq y_{2} \geq . \quad . \quad \geq y_{m} \geq . . \quad \geq y_{n-1} \geq y_{n}
$$

denotes the order statisties, that is, the observations in a sample of $n$ arranged in decreasing order of magnitude. The quantity $C=0.57721566$. . . is Euler's constant. The values $E\left(y_{m}\right)$ are tabulated herein for

$$
\begin{aligned}
& m=1(1) \min (n, 26) \\
& n=1(1) 10(5) 60(10) 100 .
\end{aligned}
$$

The table of the first moment of ranked extremes is one of the many tables furnishing pertinent information concerning the extreme-value distribution of type I. At one time Kimball [2] advocated use of such values in plotting extreme-value data on a special-scale extreme-value plotting paper. Because in this method the largest sample values are important, computations have not been carried beyond the 26th largest value $(m=26)$ for sample sizes $n>26$. Subsequent investigation [3] indicated other criteria that should be given consideration, such as the shortest confidence interval of fixed probability, which applies to the plotting position.

*Present address, David Taylor Model Basin, Washington, D. C.
**Present address, Convair-Astronauties, San Diego, Calif.

${ }_{1}^{1}$ Figures in brackets indicate the literature references at the end of this paper.
However, there arise other situations in which it is important to know where these mean values $E\left(y_{m}\right)$ are located, and with the great increase in application of the extreme-value distribution this trble should be a valuable asset.

A recent important use relates to the determination of best linear unbiased estimators for the parameters $u, \beta$ of the distribution of largest values ${ }^{2}$ in general form

$$
\text { Prob }\{X \leq x\}=F(x)=\exp \left(-e^{-y}\right), \quad y=(x-u) / \beta,
$$

or in general, for estimators of a given linear combination such as $u+\alpha \beta$ with $a$ known. The condition of unbiasedness involves a knowledge of the means, $E\left(y_{m}\right)$, of the order statistics in samples from the reduced distribution $\Phi(y)$. These are provided by the present table. The second condition, "best" i. e., minimum variance subject to the first condition, involves in addition the variances and covariances of the order statistics $\sigma^{2}\left(y_{m}\right), \sigma\left(y_{m} y_{p}\right)$.

This problem has been treated by Lieblein [4], and numerical results up to $n=6$ appear in a paper by Lieblein and Zelen [5].

\section{Interpolation Chart ${ }^{3}$ for $E\left(y_{m}\right)$}

In order to furnish values of $E\left(y_{m}\right)$ for samples of sizes not included in the table an interpolation chart designed by B. F. Kimball is appended (ing. 1).

The curves in the chart connect points having the same ranking. Thus, the lowest curve is for the expected values of the largest in a sample of size $n$; the second lowest curve relates to the second largest, etc. Each curve has two branches that meet at a vertex situated at the expected value of the median for an odd sample size. These vertices are connected by a nearly vertical line, and it is interesting to note how rapidly they approach the population median $-\ln (-\ln 0.50)=0.3665 \ldots$ as sample size $n$ increases 2 The scale parameter $\beta$ is the same as the parameter $1 / \alpha$ used in reference [1].
${ }_{3}$ This section has been contributed by B. F. Kimball. 


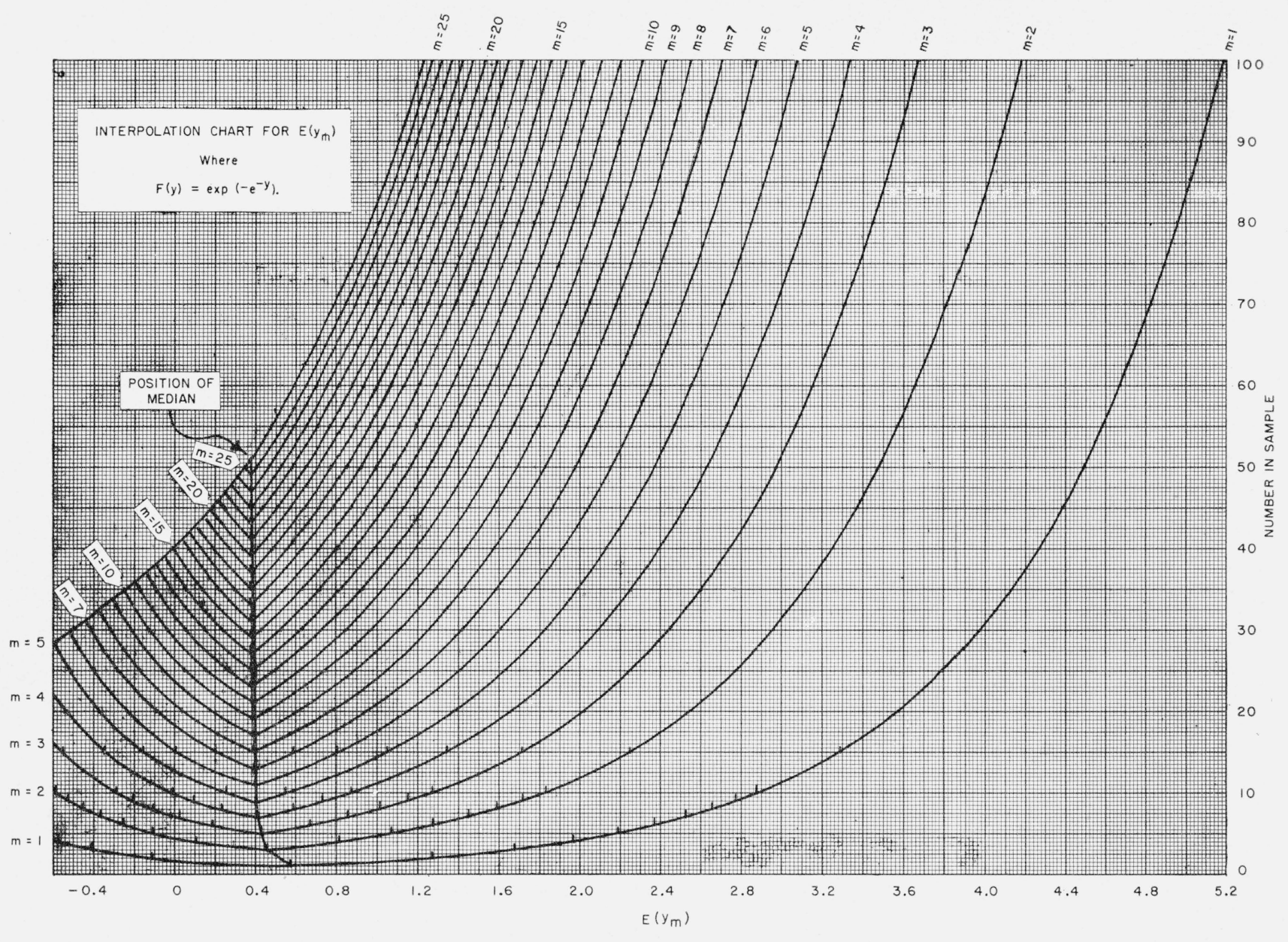

FIGURE 1.

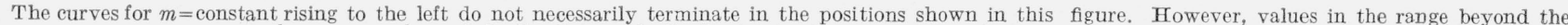
curves as shown are rarely needed. In other words, the line joining these apparent end-points might better have been omitted.

For samples of $n$ not in the table, the interpolation chart may be used mainly for finding the expected values for the ranks in the upper half of the sample. For example, let $n=75$. Then the intersections of the horizontal line $n=75$ with the curves $m=1$, $m=2, \ldots$, are read from the horizontal scale at the bottom as follows:

\begin{tabular}{|c|c|}
\hline \multicolumn{2}{|c|}{$n=75$} \\
\hline$m$ & $E\left(y_{m}\right)$ \\
\hline 1 & 4.90 \\
\hline 2 & 3.88 \\
\hline 3 & 3.38 \\
\hline 4 & 3.04 \\
\hline - & . \\
\hline . & • \\
\hline & · \\
\hline 26 & 0.88 \\
\hline
\end{tabular}

\section{Method of Computation}

Logarithms of $N$ from 1 to 120 to 48 decimal places from J. Wolfram's table [6] were punched on cards. The successive differences of the logarithms were then obtained with the aid of the IBM CPC-I. Sufficient differences were taken to attest to the accuracy of the functions and the individual differences. Values of the binomial coefficients were obtained from Peters and Stein's table [7], and the computation of $E\left(y_{m}\right)$ from eq (1) was then performed using desk calculators. The following partial check was then applied for $n \leq 25$ :

$$
\frac{1}{n} \sum_{m=1}^{n} E\left(y_{m}\right)=C
$$

For $n>25$ a differencing check was applied. The results are believed to be correct to within 2 units in the last significant figure. 
TABLE 1

$E\left(y_{m}\right)=\underset{t=0}{\substack{m-1 \\ \sum}}(-1)^{t}\left(\begin{array}{c}n \\ t !\end{array}\right) \Delta^{t} \ln (n-t)$, where $C=$ Euler's constant $=0.57721566 \ldots$

\begin{tabular}{|c|c|c|c|c|}
\hline $\begin{array}{c}\text { Rank from } \\
\text { top } \\
m\end{array}$ & $n=1$ & $n=2$ & $n=3$ & $\begin{array}{c}\text { ¿Rank from } \\
\text { top } \\
m\end{array}$ \\
\hline $\begin{array}{l}1 \\
2 \\
3\end{array}$ & 0.57721566 & $\begin{array}{l}+1.2703628 \\
-0.1159315\end{array}$ & $\begin{array}{r}1.6758280 \\
+0.4594326 \\
-0.4035136\end{array}$ & $\begin{array}{l}1 \\
2 \\
3\end{array}$ \\
\hline$m$ & $n=4$ & $n=5$ & $n=6$ & $m$ \\
\hline $\begin{array}{l}1 \\
2 \\
3 \\
4 \\
5 \\
6 \\
\end{array}$ & $\begin{array}{r}1.9635100 \\
0.8127817 \\
+.1050835 \\
-.5735126\end{array}$ & $\begin{array}{r}2.1866536 \\
1.0709358 \\
+0.4255506 \\
-.1068945 \\
-.6901671\end{array}$ & $\begin{array}{r}2.3689751 \\
1.2750458 \\
0.6627159 \\
+.1883853 \\
-.2545345 \\
-.7772937\end{array}$ & $\begin{array}{l}1 \\
2 \\
3 \\
4 \\
5 \\
5 \\
6\end{array}$ \\
\hline$m$ & $n=7$ & $n=8$ & $n=9$ & $m$ \\
\hline $\begin{array}{l}1 \\
2 \\
3 \\
4 \\
5\end{array}$ & $\begin{array}{r}2.5231258 \\
1.4440711 \\
0.8524826 \\
.4096935 \\
+.0224042\end{array}$ & $\begin{array}{r}2.6566572 \\
1.5884051 \\
1.0110660 \\
0.5881770 \\
+.2312101\end{array}$ & $\begin{array}{l}\text { 2. } 7744402 \\
\text { 1. } 7143929 \\
\text { 1. } 1474521 \\
0.7382939 \\
.4005308\end{array}$ & $\begin{array}{l}1 \\
2 \\
3 \\
4 \\
5\end{array}$ \\
\hline $\begin{array}{l}6 \\
7 \\
8 \\
9\end{array}$ & $\begin{array}{l}-.3653099 \\
-.8459576\end{array}$ & $\begin{array}{l}-.1028793 \\
-.4527868 \\
-.9021249\end{array}$ & $\begin{array}{l}+.0957534 \\
-.2021957 \\
-.5243843 \\
-.9493425\end{array}$ & $\begin{array}{l}5 \\
7 \\
8 \\
9\end{array}$ \\
\hline$m$ & $n=10$ & $n=15$ & $n=20$ & $m$ \\
\hline $\begin{array}{l}1 \\
2 \\
3 \\
4 \\
5\end{array}$ & $\begin{array}{r}2.8798008 \\
1.8261956 \\
1.2671822 \\
0.8689818 \\
.5436122\end{array}$ & 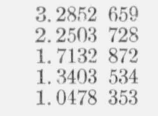 & 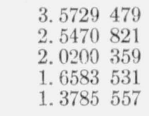 & $\begin{array}{l}1 \\
2 \\
3 \\
4 \\
5\end{array}$ \\
\hline $\begin{array}{r}6 \\
7 \\
8 \\
9 \\
10\end{array}$ & $\begin{array}{l}+.2574495 \\
-.0120439 \\
-.2836893 \\
-.5845581 \\
-.9898741\end{array}$ & $\begin{array}{r}0.8018873 \\
.5851754 \\
.3872821 \\
.2010161 \\
+.0206140\end{array}$ & $\begin{array}{r}1.1471396 \\
0.9472338 \\
.7690671 \\
.6063833 \\
.4548347\end{array}$ & $\begin{array}{r}6 \\
7 \\
8 \\
9 \\
10\end{array}$ \\
\hline $\begin{array}{l}11 \\
12 \\
13 \\
14 \\
15\end{array}$ & & $\begin{array}{r}-.1594579 \\
-.3458052 \\
-.5485327 \\
-.7883747 \\
-1.1326841\end{array}$ & $\begin{array}{r}.3111607 \\
.1727124 \\
+.0371321 \\
-.0979135 \\
-.2350333\end{array}$ & $\begin{array}{l}11 \\
12 \\
13 \\
14 \\
15\end{array}$ \\
\hline $\begin{array}{l}16 \\
17 \\
18 \\
19 \\
20\end{array}$ & & & $\begin{array}{r}-.3775607 \\
-.5304220 \\
-.7022321 \\
-.9119529 \\
-1.2232104\end{array}$ & $\begin{array}{l}16 \\
17 \\
18 \\
19 \\
20\end{array}$ \\
\hline$m$ & $n=25$ & $n=30$ & $n=35$ & $m$ \\
\hline $\begin{array}{l}1 \\
2 \\
3 \\
4 \\
5\end{array}$ & 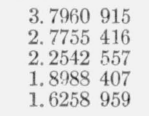 & 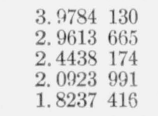 & 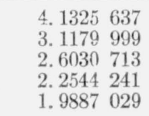 & $\begin{array}{l}1 \\
2 \\
3 \\
4 \\
5\end{array}$ \\
\hline $\begin{array}{r}6 \\
7 \\
8 \\
9 \\
10\end{array}$ & $\begin{array}{r}1.4020114 \\
1.2104327 \\
1.0415346 \\
0.8892472 \\
.7494644\end{array}$ & $\begin{array}{l}1.6044708 \\
1.4178745 \\
1.2543779 \\
1.1079713 \\
0.9746216\end{array}$ & 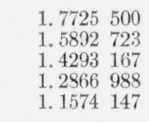 & $\begin{array}{r}6 \\
7 \\
8 \\
9 \\
10\end{array}$ \\
\hline $\begin{array}{l}11 \\
12 \\
13 \\
14 \\
15\end{array}$ & $\begin{array}{l}.6192453 \\
.4963746 \\
.3791010 \\
.2559691 \\
.1557008\end{array}$ & 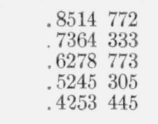 & $\begin{array}{r}1.0386471 \\
0.9283319 \\
.8249044 \\
.7271437 \\
.6340712\end{array}$ & $\begin{array}{l}11 \\
12 \\
13 \\
14 \\
15\end{array}$ \\
\hline $\begin{array}{l}16 \\
17 \\
18 \\
19 \\
20\end{array}$ & $\begin{array}{l}+.0471025 \\
-.0610213 \\
-.1699655 \\
-.2812545 \\
-.3958504\end{array}$ & $\begin{array}{r}.3294297 \\
.2360031 \\
.1443471 \\
+.0537740 \\
-.0364087\end{array}$ & $\begin{array}{l}.5448828 \\
.4589015 \\
.3755427 \\
.2942883 \\
.2146659\end{array}$ & $\begin{array}{l}16 \\
17 \\
18 \\
19 \\
20\end{array}$ \\
\hline $\begin{array}{l}21 \\
22 \\
23 \\
24 \\
25\end{array}$ & $\begin{array}{r}-.5195330 \\
-.6536981 \\
-.8073485 \\
-.9984861 \\
-1.2882598\end{array}$ & $\begin{array}{l}-.1269375 \\
-.2186413 \\
-.3125115 \\
-.4098117 \\
-.5122664\end{array}$ & $\begin{array}{r}.1362310 \\
+.0585513 \\
-.0188098 \\
-.0963110 \\
-.1744547\end{array}$ & $\begin{array}{l}21 \\
22 \\
23 \\
24 \\
25\end{array}$ \\
\hline 26 & & -.6224196 & -.2538157 & 26 \\
\hline$m$ & $n=40$ & $n=45$ & $n=50$ & $m$ \\
\hline $\begin{array}{l}1 \\
2 \\
3 \\
4 \\
5\end{array}$ & 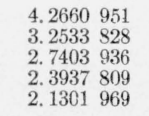 & $\begin{array}{ll}4.3838 & 782 \\
3.3725 & 996 \\
2.8611 & 039 \\
2.5160 & 483 \\
2.2540 & 897\end{array}$ & $\begin{array}{ll}\text { 4. } 4892 & 387 \\
3.4791 & 033 \\
2.9687 & 929 \\
2.6249 & 676 \\
2.3542 & 868\end{array}$ & $\begin{array}{l}1 \\
2 \\
3 \\
4 \\
5\end{array}$ \\
\hline
\end{tabular}

\begin{tabular}{|c|c|c|c|c|}
\hline $\begin{array}{c}\text { Rank from } \\
\text { top } \\
m\end{array}$ & $n=40$ & $n=45$ & $n=50$ & $\begin{array}{c}\text { Rank from } \\
\text { top } \\
m\end{array}$ \\
\hline $\begin{array}{r}6 \\
7 \\
8 \\
9 \\
10\end{array}$ & $\begin{array}{l}\text { 1. } 9162926 \\
\text { 1. } 7353 \quad 848 \\
\text { 1. } 5779311 \\
\text { 1. } 4379596 \\
\text { 1. } 3114804\end{array}$ & 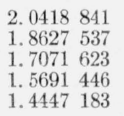 & 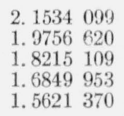 & $\begin{array}{r}6 \\
7 \\
8 \\
9 \\
10\end{array}$ \\
\hline $\begin{array}{l}11 \\
12 \\
13 \\
14 \\
15\end{array}$ & $\begin{array}{r}1.1956921 \\
1.0885487 \\
0.9885067 \\
.8943683 \\
.8051863\end{array}$ & $\begin{array}{l}\text { 1. } 3310904 \\
\text { 1, 2262 } 243 \\
\text { 1. } 1285871 \\
\text { 1. } 0369939 \\
0.9505080\end{array}$ & $\begin{array}{l}1.4501482 \\
1.3469974 \\
1.2511576 \\
1.1614507 \\
1.0769470\end{array}$ & $\begin{array}{l}11 \\
12 \\
13 \\
14 \\
15\end{array}$ \\
\hline $\begin{array}{l}16 \\
17 \\
18 \\
19 \\
20\end{array}$ & $\begin{array}{l}.7201865 \\
.6387319 \\
.5602845 \\
.4843816 \\
.4106 \\
182\end{array}$ & $\begin{array}{l}.8683738 \\
.7899709 \\
.7147813 \\
.6423661 \\
.5723476\end{array}$ & $\begin{array}{r}0.9968991 \\
.9206958 \\
.8478294 \\
.7778727 \\
.7104609\end{array}$ & $\begin{array}{l}16 \\
17 \\
18 \\
19 \\
20\end{array}$ \\
\hline $\begin{array}{l}21 \\
22 \\
23 \\
24 \\
25\end{array}$ & $\begin{array}{r}.3386325 \\
.2680942 \\
.1986951 \\
.1301405 \\
+.0621408\end{array}$ & $\begin{array}{l}.5043959 \\
.4382186 \\
.3735525 \\
.3101567 \\
.2478069\end{array}$ & $\begin{array}{l}.6452792 \\
.5820524 \\
.5205370 \\
.4505148 \\
.4017883\end{array}$ & $\begin{array}{l}21 \\
22 \\
23 \\
24 \\
25\end{array}$ \\
\hline 26 & -.0055967 & .1862904 & .3441759 & 26 \\
\hline$m$ & $n=55$ & $n=60$ & $n=70$ & $m$ \\
\hline $\begin{array}{l}1 \\
2 \\
3 \\
4 \\
5\end{array}$ & 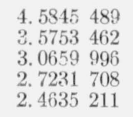 & 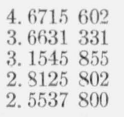 & 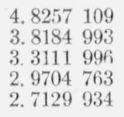 & $\begin{array}{l}1 \\
2 \\
3 \\
4 \\
5\end{array}$ \\
\hline $\begin{array}{r}6 \\
7 \\
8 \\
9 \\
10\end{array}$ & 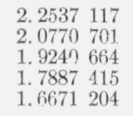 & 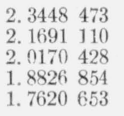 & 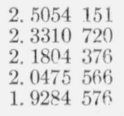 & $\begin{array}{r}6 \\
7 \\
8 \\
9 \\
10\end{array}$ \\
\hline $\begin{array}{l}11 \\
12 \\
13 \\
14 \\
15\end{array}$ & 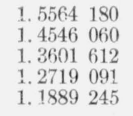 & 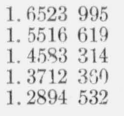 & $\begin{array}{l}\text { 1. } 8203598 \\
\text { 1. } 7212394 \\
\text { 1. } 6295 \\
\text { 1. } 5442060 \\
\text { 1. } 4642047\end{array}$ & $\begin{array}{l}11 \\
12 \\
13 \\
14 \\
15\end{array}$ \\
\hline $\begin{array}{l}16 \\
17 \\
18 \\
19 \\
20\end{array}$ & $\begin{array}{r}1.1104647 \\
1.0359236 \\
0.9647993 \\
.8966708 \\
.8311807\end{array}$ & 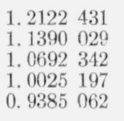 & 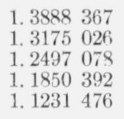 & $\begin{array}{l}16 \\
17 \\
18 \\
19 \\
20\end{array}$ \\
\hline $\begin{array}{l}21 \\
22 \\
23 \\
24 \\
25\end{array}$ & $\begin{array}{l}.7680221 \\
.7069285 \\
.6476664 \\
.5900293 \\
.5338321\end{array}$ & $\begin{array}{l}.8768913 \\
.8174137 \\
.7598456 \\
.7039865 \\
.6496584\end{array}$ & $\begin{array}{r}\text { 1. } 0637356 \\
1.0065469 \\
0.9513592 \\
.8979781 \\
.8462324\end{array}$ & $\begin{array}{l}21 \\
22 \\
23 \\
24 \\
25\end{array}$ \\
\hline 26 & .4789079 & .5967022 & 7959700 & 26 \\
\hline$m$ & $n=80$ & $n=90$ & $n=100$ & $m$ \\
\hline $\begin{array}{l}1 \\
2 \\
3 \\
4 \\
5\end{array}$ & 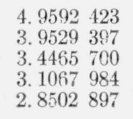 & 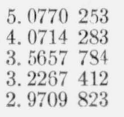 & 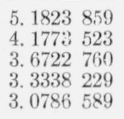 & $\begin{array}{l}1 \\
2 \\
3 \\
4 \\
5\end{array}$ \\
\hline $\begin{array}{r}6 \\
7 \\
8 \\
9 \\
10\end{array}$ & $\begin{array}{l}2.6437091 \\
2.4703 \quad 881 \\
2.3208011 \\
2.1989938 \\
2.0709961\end{array}$ & 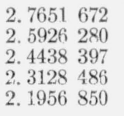 & 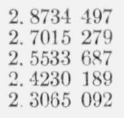 & $\begin{array}{r}6 \\
7 \\
8 \\
9 \\
10\end{array}$ \\
\hline $\begin{array}{l}11 \\
12 \\
13 \\
14 \\
15\end{array}$ & $\begin{array}{l}1.9640281 \\
1.8660674 \\
1.7755969 \\
1.6914486 \\
1.6127044\end{array}$ & $\begin{array}{l}\text { 2. } 0895790 \\
\text { 1. } 9924818 \\
\text { 1. } 9029038 \\
\text { 1. } 8196690 \\
1.7418600\end{array}$ & 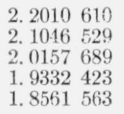 & $\begin{array}{l}11 \\
12 \\
13 \\
14 \\
15\end{array}$ \\
\hline $\begin{array}{l}16 \\
17 \\
18 \\
19 \\
20\end{array}$ & 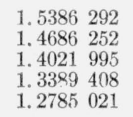 & 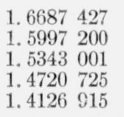 & $\begin{array}{l}\text { 1. } 7837772 \\
1.7155086 \\
1.5508589 \\
1.5894186 \\
1.5308422\end{array}$ & $\begin{array}{l}16 \\
17 \\
18 \\
19 \\
20\end{array}$ \\
\hline $\begin{array}{l}21 \\
22 \\
23 \\
24 \\
25\end{array}$ & 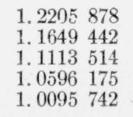 & 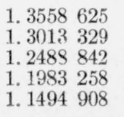 & 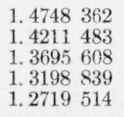 & $\begin{array}{l}21 \\
22 \\
23 \\
24 \\
25\end{array}$ \\
\hline 26 & 0.9610726 & 1. 1022318 & 1. 2256168 & 26 \\
\hline
\end{tabular}


The calculations were done in the Computation Laboratory of the National Bureau of Standards. Computations were performed under the supervision of Herbert E. Salzer. The inal checking was carried out under the direction of Irene Stegun.

\section{References}

[1] Statistical theory of extreme values and some practical applications, NBS Appl. Math. Series 33 (1954). See especially lecture 3, p. 19-21, and, for a summary of the characteristics of the distribution, table 3.1 on p. 24-25. For a companion volume of tables see Probability tables for the analysis of extreme-value data, NBS Appl. Math. Series 22 (1953).

[2] B. F. Kimball, Assignment of frequencies to a completely ordered set of sample data, Trans. Am. Geophysical Union 2\%, 843-846 (1946). See also discussion of this article in $\mathbf{2 8 , 9 5 1 - 9 5 3 ~ ( 1 9 4 7 ) . ~}$
[3] B. F. Kimball, An aid to judging precision and bias of graphical fit to data plotted on normal or extreme-value plotting paper, (submitted for publication).

[4] J. Lieblein, On the exact evaluation of the variances and covariances of order statistics in samples from the extreme-value distribution, Ann. Math. Stat. 24, 282-287 (1953)

[5] J. Lieblein and M. Zelen, Statistical investigation of the fatigue life of deep-groove ball bearings, J. Research NBS 5\%, 273-316 (1956) RP2719.

[6] J. Wolfram, Tabula logarithmorum naturalium, p. 641-684 of G. von Vega, Thesaurus logarithmorum completus, Leipzig, 1794 (reprints published by Stechert Hafner, Inc., New York, N. Y., 1923)

[7] J. Peters and J. Stein, Part of appendix to Peters' Zehnstellige Logarithmentafel, Band I (Berlin, 1922).

Washington, February 26, 1957. 